\title{
Combination of cytokinin and auxin induces apoptosis, cell cycle progression arrest and blockage of the Akt pathway in HeLa cells
}

\author{
LIWEI ZHAO, PENG LIU, GUANGQIN GUO and LI WANG \\ Department of Cell Biology, School of Life Sciences, Lanzhou University, Lanzhou 730000, P.R. China
}

Received April 11, 2014; Accepted January 15, 2015

DOI: $10.3892 / \mathrm{mmr} .2015 .3420$

\begin{abstract}
Plant cytokinins and auxins have recently been proposed as novel cancer therapies, which proceed via different mechanisms; however, their combined use has not been investigated. To the best of our knowledge, the present study was the first to show that the cytokinin ortho-methoxytopolin-riboside (MeoTR) strongly inhibited the proliferation of HeLa cells, the effect of which was synergistically enhanced by auxin indole-3-acetic acid (IAA), while IAA demonstrated to have no cytotoxic effects on cells. MeoTR was found to activate intrinsic and extrinsic caspase-dependent pathways, and IAA potentiated this activation. In addition, these effects were blocked by Z-Val-Ala-Asp-fluoromethylketone (Z-VAD-FM $\mathrm{K}$ ), a pan-specific-caspase-inhibitor. IAA increased the MeoTRinduced inhibition of B cell lymphoma 2 (Bcl-2) and survivin, whereas IAA-only decreased Bcl-2 expression. MeoTR downregulated phosphorylated (p)-pyruvate dehydrogenase kinase 1 , p-Akt and p-glycogen synthase kinase $3 \beta$, the effect of which was more potent in combination with IAA, despite the weak effect of IAA alone. LY294002, an Akt-inhibitor, was able to increase the inhibition of p-Akt through MeoTR and combination treatment. IAA and MeoTR increased the proportion of cells in S phase independently. However, the combination treatment induced a further increase. In addition, IAA and MeoTR treatment downregulated protein levels of cyclin A, cyclin-dependent kinase 2 (CDK2) and p-CDK2, and upregulated protein levels of p21 and p27. Furthermore, the combination treatment enhanced these effects, indicating that IAA potentiated the inhibitory effect of MeoTR on HeLa cells via cell cycle progression arrest and accumulation in $\mathrm{S}$ phase, coupled with the negative regulation of $\mathrm{Bcl}-2$. In conclusion, the results of the present study suggested that treatment with these
\end{abstract}

Correspondence to: Professor Guangqin Guo and Dr Li Wang, Department of Cell Biology, School of Life Sciences, Lanzhou University, 222 Tian Shui Road, Chengguan, Lanzhou 730000, P.R. China

E-mail: gqguo@lzu.edu.cn

E-mail: wang_li@dlut.edu.cn

Key words: ortho-methoxytopolin riboside, indole-3-acetic acid, combination, apoptosis, cell cycle progression arrest, Akt pathway two phytohormones in combination, may offer a novel therapeutic strategy for the treatment of malignant cervical cancer.

\section{Introduction}

Cytokinins are critical hormones for plant growth and development. Historically, they were defined by their ability to promote cell division when combined with auxin in tobacco tissue culture (1). Further studies demonstrated that cytokinins were involved in several processes in plants (2). Chemically, cytokinins are $\mathrm{N}^{6}$-substituted adenine derivatives (3). Cytokinins are divided into three categories, according to their substituent, as follows: Isoprenoid cytokinins, including isopentenyladenine, zeatin and zeatin riboside; furfural derivatives, including kinetin and kinetin riboside; and aromatic cytokinins, including ortho-topolin riboside (oTR), ortho-methoxytopolin-riboside (MeoTR) and $\mathrm{N}^{6}$-benzyladenosine (4).

Since the first aromatic cytokinin oTR was isolated from mature leaves of poplar (5), aromatic cytokinins have also been detected in oil palm (6) and microalgae (7). Previous studies have shown that aromatic cytokinins exhibit toxicity against different types of human cancer cells and their effectiveness in the treatment of tumors was reported to be mediated through inhibition of cell proliferation and/or differentiation (8-11). $\mathrm{N}^{6}$-benzyladenosine was found to induce cell cycle arrest and apoptosis in bladder carcinoma T24 cells (12). A previous study revealed that oTR had a strong apoptosis-inducing effect on the human hepatoma SMMC-7721 cell line (11). MeoTR and oTR share an identical parental structure, except for the substitution of the hydroxyl in benzene with a methoxy group. However, the effect of MeoTR on human cancer cells has not yet been studied.

Indole-3-acetic acid (IAA) is a naturally present auxin and a vital regulator of plant cell division, elongation and differentiation (13). Previous studies have shown that IAA affected several different mammalian cell functions; IAA, activated by horseradish peroxidase (HRP) $(14,15)$ or intense pulsed light $(16,17)$, was found to exhibit potent toxicity against cancer cells, including G361 human melanoma cells (14) and PC-3 prostate cancer cells (18). However, the effect of IAA treatment alone against tested cancer cells did not produce significant results $(14,17,18)$. Furthermore, to the best of our knowledge, a comparative and systematic study of the effects of a combination treatment with cytokinin and auxin on human cancer cells has yet to be performed. 
The aim of the present study was to investigate the inhibitory effects of MeoTR and/or IAA in the human cervical cancer HeLa cell line. We showed that treatment with MeoTR alone arrested cell cycle progression, and accumulated in $\mathrm{S}$ phase, and induced apoptosis via intrinsic and extrinsic caspase-dependent pathways in vitro. These effects were enhanced by combination treatment with IAA. Although IAA did not affect cell proliferation, it induced significant cell accumulation in $\mathrm{S}$ phase, indicating that its potentiating effect on MeoTR may be mediated by its cytostatic rather than cytotoxic activity. MeoTR treatment blocked the Akt pathway, and these effects were enhanced by IAA, although IAA had no effect on the Akt pathway when used alone.

\section{Materials and methods}

Reagents. MeoTR was purchased from OlChemIm Ltd. (Olomouc, Czech Republic) and IAA, Z-Val-Ala-Asp-fluo romethylketone (Z-VAD-FMK), LY294002 and violet staining solution were purchased from Sigma-Aldrich (St. Louis, MO, USA). Insulin-like growth factor I (IGF-I), an Akt activation factor, was purchased from PeproTech, Inc. (Rocky Hill, CT, USA). The maximum final concentration of dimethyl sulfoxide (DMSO) in the culture medium was $<0.5 \%$. XTT and phenazine methosulfate (PMS) were purchased from Sangon Biotech Co., Ltd (Shanghai, China). All additional solid reagents were purchased from Sigma-Aldrich. The following primary antibodies: Monoclonal anti-cleaved poly-adenosine diphosphate-ribose polymerase (PARP; cat. no. 5625), polyclonal anti-total PARP (cat. no. 9542), monoclonal anti-cytochrome $c$ (cat. no. 11940), monoclonal anti-B cell lymphoma 2 (Bcl-2; cat. no. 2870), monoclonal anti-survivin (cat. no. 2808), monoclonal anti-phosphorylated (p)-pyruvate dehydrogenase kinase 1 (PDK1; cat. no. 3438), monoclonal anti-p-Akt (Ser473; cat. no. 4060), monoclonal anti-Akt (cat. no. 4685), monclonal anti-p-glycogen synthase kinase $3 \beta$ (GSK-3 $\beta$; cat. no. 5558), monoclonal anti-GSK-3 $\beta$ (cat. no. 12456) and monoclonal anti- $\beta$-actin (cat. no. 3700), were purchased from Cell Signaling Technology, Inc. (Beverly, MA, USA). The following primary antibodies: Polyclonal anti-cyclin A (cat. no. sc-751), polyclonal anti-p-cyclin-dependent kinase 2 (CDK2) (Thr160) (cat. no. sc-101656), polyclonal anti-CDK2 (cat. no. sc-163), polyclonal anti-p21 (cat. no sc-397) and polyclonal anti-p27 (cat. no. sc-528), were purchased from Santa Cruz Biotechnology, Inc. (Dallas, TX, USA) and primary antibodies, polyclonal anti-caspase-3 (cat. no. 29010), polyclonal anti-caspase-9 (cat. no. 22978) and polyclonal anti-caspase-8 (cat. no. 22977) were purchased from Signalway Antibody (College Park, MD, USA). Goat anti-mouse (\#7072) and goat anti-rabbit (\#7071) secondary IgG antibodies were purchased from Cell Signaling Technology, Inc.

Cell culture. Human cervical cancer HeLa cells (Cell Bank of the Chinese Academy of Sciences, Shanghai, China) were cultured in RPMI-1640 medium supplemented with $10 \%$ fetal bovine serum, $100 \mu \mathrm{g} / \mathrm{ml}$ penicillin and $100 \mu \mathrm{g} / \mathrm{ml}$ streptomycin in a humidified atmosphere containing $5 \% \mathrm{CO}_{2}$ at $37^{\circ} \mathrm{C}$.

Cell viability assay. Vehicle (0.4\% DMSO)-treated cells were considered the control cells. Cell viability was determined using the XTT assay. HeLa cells were seeded in 96-well plates at a density of $5.0 \times 10^{4}$ cells/well for $12 \mathrm{~h}$ prior to treatment. For the dose-dependent assay, solutions containing various concentrations of MeoTR $(25,50,75$ and $100 \mu \mathrm{M})$ and IAA $(20,50,100,500$ and $1000 \mu \mathrm{M})$, independently, or MeoTR $(25,50,75$ and $100 \mu \mathrm{M})$ in combination with $1 \mathrm{mM}$ IAA were added to each well in $100 \mu \mathrm{l}$ RPMI-1640 medium and incubated for $48 \mathrm{~h}$. For the time course assay, solutions containing $50 \mu \mathrm{M}$ MeoTR and $1 \mathrm{mM}$ IAA, alone or in combination, were added to each well in $100 \mu \mathrm{l}$ RPMI-1640 medium and incubated for $0,12,24$ and $48 \mathrm{~h}$. Following treatment, $50 \mu \mathrm{l}$ XTT solution ( $1 \mathrm{~g} / 1 \mathrm{XTT}$ and $5 \mathrm{mg} / \mathrm{l} \mathrm{PMS}$ in RPMI-1640 medium) was added to each well for a further $4 \mathrm{~h}$ at $37^{\circ} \mathrm{C}$. Absorbance was read at $595 \mathrm{~nm}$ using a microplate reader (Thermo Electron Corporation, Waltham, MA, USA). Three independent experiments were performed from six replicates of each treatment group. The percentage cell viability was calculated as follows: [sample optical density (OD)/control OD] x100\%.

The coefficient of drug interaction (CDI) was used to analyze the synergistically inhibitory effect of drug combinations. $\mathrm{CDI}=\mathrm{AB} /(\mathrm{AxB})$, where $\mathrm{AB}$ represents the cell viability of the combination group and A or B represent the cell viabilities of the single agent groups, as determined by measuring the absorbance of each group. $\mathrm{CDI}<1$ indicates that the drugs are synergistic; $\mathrm{CDI}=1$ indicates that the drugs are additive; CDI $>1$ indicates that the drugs are antagonistic; in addition, CDI $<0.7$ indicates that the drugs are significantly synergistic, as previously described (19).

Crystal violet staining. HeLa cells were seeded in 6-well plates at a density of $5.0 \times 10^{4}$ cells/well for $12 \mathrm{~h}$. The cells were treated with MeoTR (50 and $100 \mu \mathrm{M})$ and/or $1 \mathrm{mM}$ IAA for a further $48 \mathrm{~h}$. Cells were then fixed with $4 \%$ paraformaldehyde and stained with crystal violet staining solution. The cell morphology was observed using a Nikon Eclipse Ti-U Multi-port inverted microscope (Nikon Corp., Tokyo, Japan).

Flow cytometric analysis of apoptosis and cell cycle. Cell apoptosis and cell cycle distribution were analyzed using flow cytometry. HeLa cells were pre-treated with MeoTR (50 and $100 \mu \mathrm{M}$ ) and/or $1 \mathrm{mM}$ IAA for $48 \mathrm{~h}$. Cell apoptosis was assessed using a fluorescein isothiocyanate-Annexin $\mathrm{V}$ apoptosis kit (BD Biosciences, San Jose, CA, USA) according to the manufacturer's instructions. The stained cells were then analyzed using a FACSCalibur flow cytometer (BD Biosciences). For cell cycle analysis, HeLa cells were harvested and washed twice with ice-cold phosphate-buffered saline (PBS), resuspended in $1 \mathrm{ml} 75 \%$ ethanol $\left(-20^{\circ} \mathrm{C}\right)$ and fixed at $-20^{\circ} \mathrm{C}$ overnight. Fixed cells were washed with PBS, treated with $50 \mu \mathrm{g} / \mathrm{ml}$ RNase A for $5 \mathrm{~min}$ at room temperature and stained with propidium iodide (PI; $50 \mu \mathrm{g} / \mathrm{ml}$ ) in the dark at $4^{\circ} \mathrm{C}$ for $30 \mathrm{~min}$. The stained cells were then assessed using a FACSCalibur flow cytometer. Each assay was performed in triplicate.

Western blotting. Following treatment with the indicated concentrations of MeoTR and/or $1 \mathrm{mM}$ IAA for $48 \mathrm{~h}$, the cells were lysed in RIPA lysis buffer. The concentration of total protein was determined using a bicinchoninic acid protein assay kit (Thermo Fisher Scientific, Waltham, MA, USA). For western blot analysis, $30 \mu \mathrm{g}$ protein was resolved using 
$\mathbf{A}$

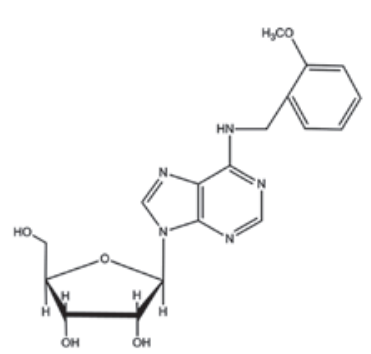

D

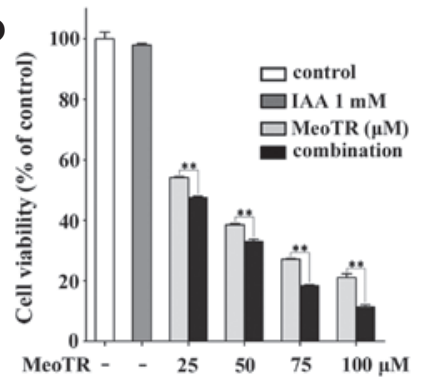

B

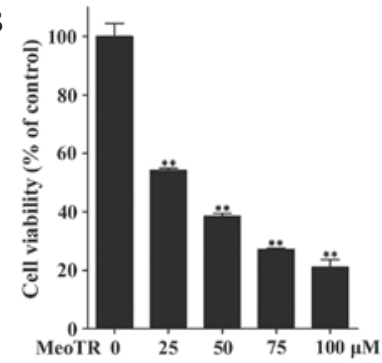

$\mathbf{E}$

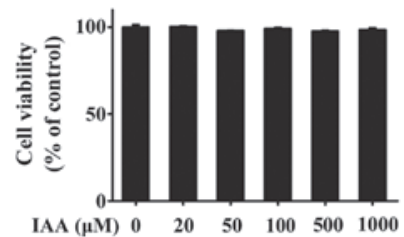

C

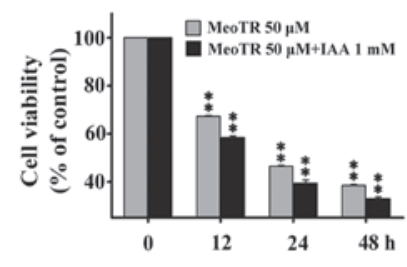

F

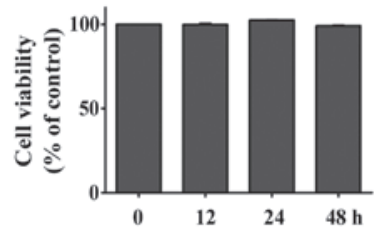

G

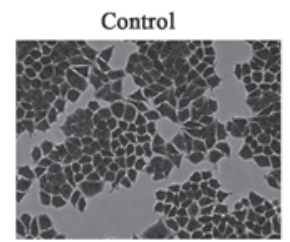

MeoTR $50 \mu \mathrm{M}$

MeoTR $50 \mu \mathrm{M}+$ IAA $1 \mathrm{mM}$
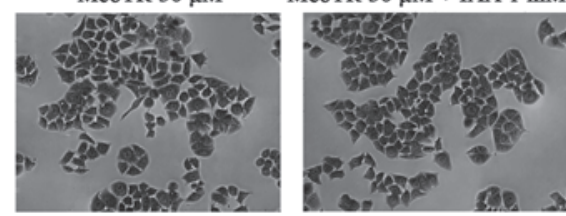

IAA $1 \mathrm{mM}$

MeoTR $100 \mu \mathrm{M}$

MeoTR $100 \mu \mathrm{M}+$ IAA $1 \mathrm{mM}$
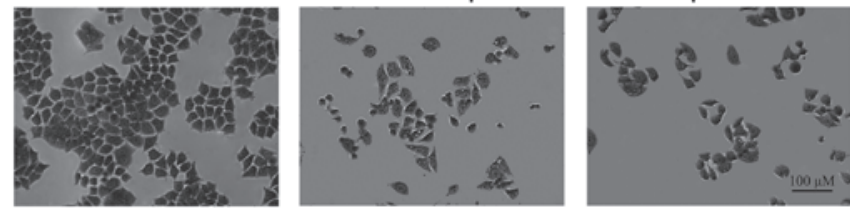

Figure 1. Cytotoxic effects of MeoTR and/or IAA on HeLa human cervical cancer cells. (A) Structure of MeoTR (molecular weight, 387.4). HeLa cells were cultured with different concentration of MeoTR and/or IAA, and cell viability was measured using the XTT assay. (B) Cell viability following treatment with MeoTR $(25,50,75$ and $100 \mu \mathrm{M})$ for $48 \mathrm{~h}$. (C) Cell viability following treatment with $50 \mu \mathrm{M}$ MeoTR and/or $1 \mathrm{mM}$ IAA for increasing incubation times (0, 12 , 24 and 48 h). (D) Cell viability following treatment with MeoTR alone or in combination with 1 mM IAA for 48 h. (E) Cell viability following treatment with IAA $(20,50,100,500$ and $1000 \mu \mathrm{M})$ alone for $48 \mathrm{~h}$. (F) Cell viability following treatment with $1 \mathrm{mM}$ IAA for increasing incubation times $(0,12,24$ and $48 \mathrm{~h})$. Values are expressed as the mean \pm standard error of the mean for three independent experiments. ${ }^{*} \mathrm{P}<0.05$ and ${ }^{* *} \mathrm{P}<0.01$ vs. vehicle-treated control cells or MeoTR-treated cells. (G) Changes in cell morphology following incubation with MeoTR alone and in combination with IAA was observed using crystal violet staining for $48 \mathrm{~h}$. MeoTR, ortho-methoxytopolin-riboside; IAA, indole-3-acetic acid.

$10 \%$ SDS-PAGE and transferred to polyvinylidene difluoride membranes. Blots were blocked in tris-buffered saline with Tween-20 (TBST), containing $0.01 \%$ Tween-20 and 5\% non-fat dry milk for $1 \mathrm{~h}$, and incubated with primary antibodies indicated above overnight at $4^{\circ} \mathrm{C}$ with agitation. Membranes were then washed five times with TBST for $10 \mathrm{~min}$ each, followed by incubation with the indicated secondary antibodies for $2 \mathrm{~h}$ at $4^{\circ} \mathrm{C}$. Membranes were washed with TBST five times (10 min each) and peroxidase activity was visualized using the enhanced chemiluminescence method. Results were quantified through grey correlation analysis of bands using Image $\mathbf{J}$ software (National Institute of Health, Bethesda, MD, USA). Assays were performed in triplicate.

Statistical analysis. Values are expressed as the mean \pm standard error of the mean for three individual experiments. Statistical significance was determined using the Student's t-test. $\mathrm{P}<0.05$ and $\mathrm{P}<0.01$ were considered to indicate statistically significant differences between values.

\section{Results}

IAA enhances the effect of MeoTR on the inhibition of HeLa cell viability. The chemical structure of MeoTR is shown in Fig. 1A. The effect of MeoTR and/or IAA on the survival of HeLa cells was assessed using an XTT assay. MeoTR alone or in combination with IAA was found to significantly decrease HeLa cell viability in dose- and time-dependent manners compared with that of the vehicle-treated control or MeoTR-treated cells, respectively $(\mathrm{P}<0.05)$ or $\mathrm{P}<0.01)$ (Fig. 1B, C and D), whereas IAA alone had no cytotoxic effects (Fig. 1E and F). MeoTR reduced HeLa cell viability to $54.1,38.5,27.1$ and $21.0 \%$ of the control values at concentrations of $25,50,75$ and $100 \mu \mathrm{M}$, respectively, and combination treatment with IAA further reduced cell viability to 47.5 , $32.9,18.3$ and $11.3 \%$ at these concentrations, respectively (Fig. 1B and D). The CDI indicated that IAA acted significantly synergistically with MeoTR (Table I). In addition, the potent growth inhibition of MeoTR alone and in combination 
Table I. Synergistically inhibitory effect of MeoTR and IAA combination treatment.

\begin{tabular}{l}
\hline \multicolumn{1}{c}{ MeoTR $(\mu \mathrm{m})$} \\
\cline { 2 - 3 } \\
\cline { 2 - 3 }
\end{tabular}
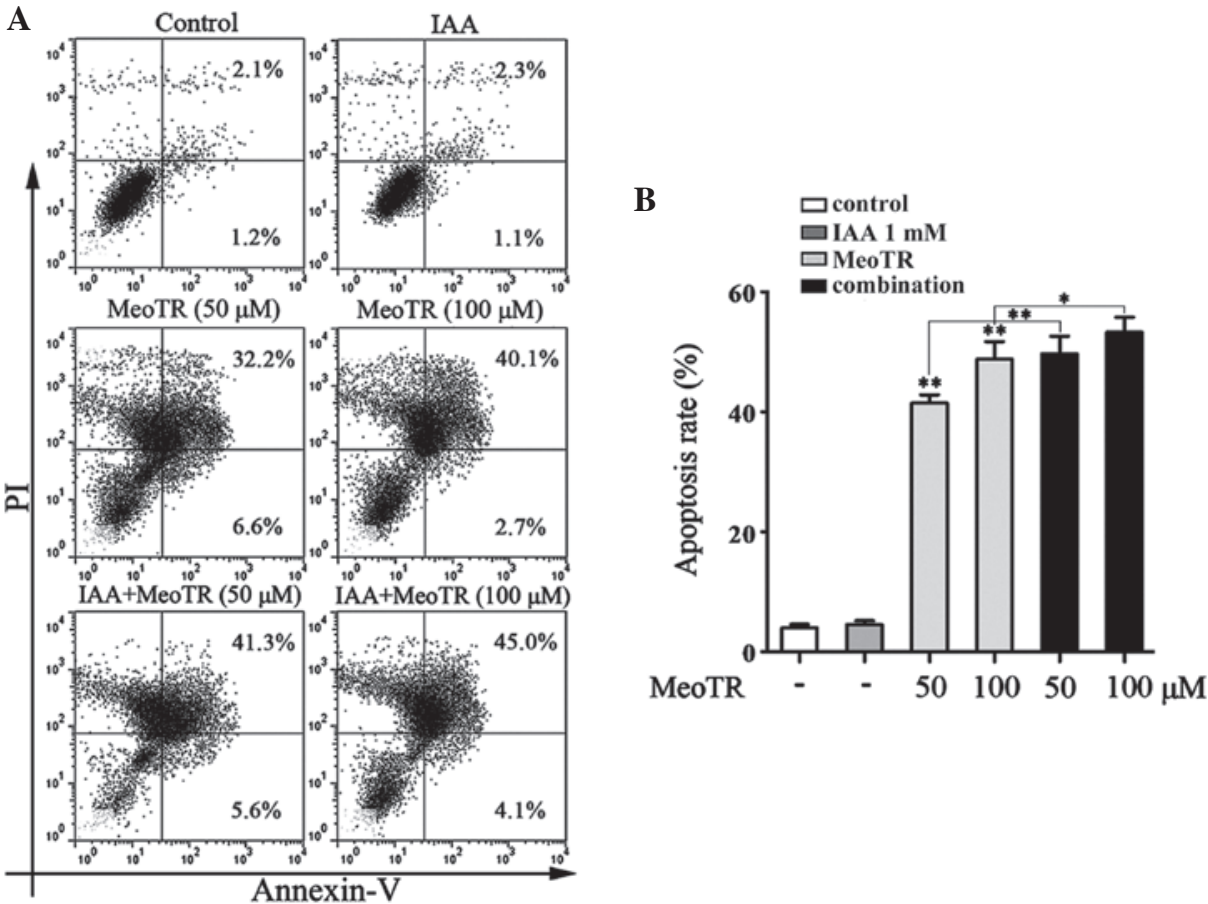

Figure 2. Effect of MeoTR and/or IAA treatment on apoptosis. (A) Apoptosis was analyzed using flow cytometry with Annexin V-fluorescein isothiocyanate and PI double staining. (B) Percentage of apoptotic cells is presented as the mean \pm standard error of the mean for three independent experiments. ${ }^{*} \mathrm{P}<0.05$ and ${ }^{* *} \mathrm{P}<0.01$ vs. vehicle-treated control cells or MeoTR-treated cells. MeoTR, ortho-methoxytopolin-riboside; IAA, indole-3-acetic acid; PI, propidium iodide.

with IAA was further confirmed in the crystal violet staining assay (Fig. 1G). Additionally, the obvious decrease of cell numbers, microscopic examination of cell morphology revealed certain major patterns of shape change in response to treatment with MeoTR alone or in combination with IAA. The treated cells were smaller, rounder and deep-stained compared with those of the control group, as well as detached from the culture well surface, indicating marked cell damage (Fig. 1G).

IAA enhances the effect of MeoTR on the induction of HeLa cell apoptosis. In order to elucidate the mechanism of MeoTR-induced cell death, the pro-apoptotic effects of MeoTR were measured using flow cytometric analysis following Annexin V/PI staining. As shown in Fig. 2, MeoTR induced cell apoptosis in a dose-dependent manner and combined treatment with IAA further enhanced this induction of apoptosis, while IAA alone was unable to induce apoptosis.
These findings indicated that apoptosis may contribute to the anti-proliferative effect of MeoTR in HeLa cells, the effect of which was enhanced in the presence of IAA.

MeoTR induces apoptosis through intrinsic and extrinsic caspase-dependent pathway and IAA potentiates this effect. In order to investigate whether caspase-dependent apoptotic pathways were activated in response to MeoTR, HeLa cells were treated with different concentrations of MeoTR and/or $1 \mathrm{mM}$ IAA for $48 \mathrm{~h}$ and then lysed for western blot analysis. The release of cytochrome $c$ from mitochondria into the cytosol has an important role in the pro-apoptotic mechanisms of the intrinsic caspase-dependent pathways (20). As shown in Fig. 3A, MeoTR treatment increased the cytosolic levels of cytochrome $\mathrm{c}$ in a dose-dependent manner, which led to the lysis of its downstream protein zymogen caspase- 9 . However, no changes in cytochrome $c$ or caspase-9 were detected following pre-treatment with IAA alone (Fig. 4A), 




C

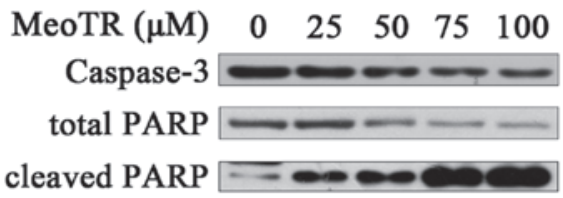

$\beta$-actin ---
B

$\begin{array}{cccccc}\text { MeoTR }(\mu \mathrm{M}) & 0 & 25 & 50 & 75 & 100 \\ \text { Caspase-8 } & & & & & \end{array}$

D

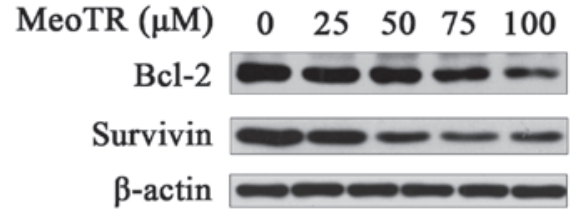

Figure 3. Effect of MeoTR on caspase-dependent apoptosis pathway biomarker protein expression in HeLa cells. HeLa cells were pretreated with MeoTR (25, $50,75$ and $100 \mu \mathrm{M})$ for $48 \mathrm{~h}$, and the lysed for western blot analysis using specific antibodies. $\beta$-actin was used as an internal protein loading control. Western blot analysis of: (A) The release of cytochrome $c$ to the cytosol from mitochondria and the activation of caspase-9, (B) effect of MeoTR on the activation of caspase-8, (C) activation levels of caspase-3 and PARP, and (D) expression of Bcl-2 and survivin. MeoTR, ortho-methoxytopolin-riboside; PARP, poly-adenosine diphosphate-ribose polymerase; Bcl-2, B cell lymphoma 2.

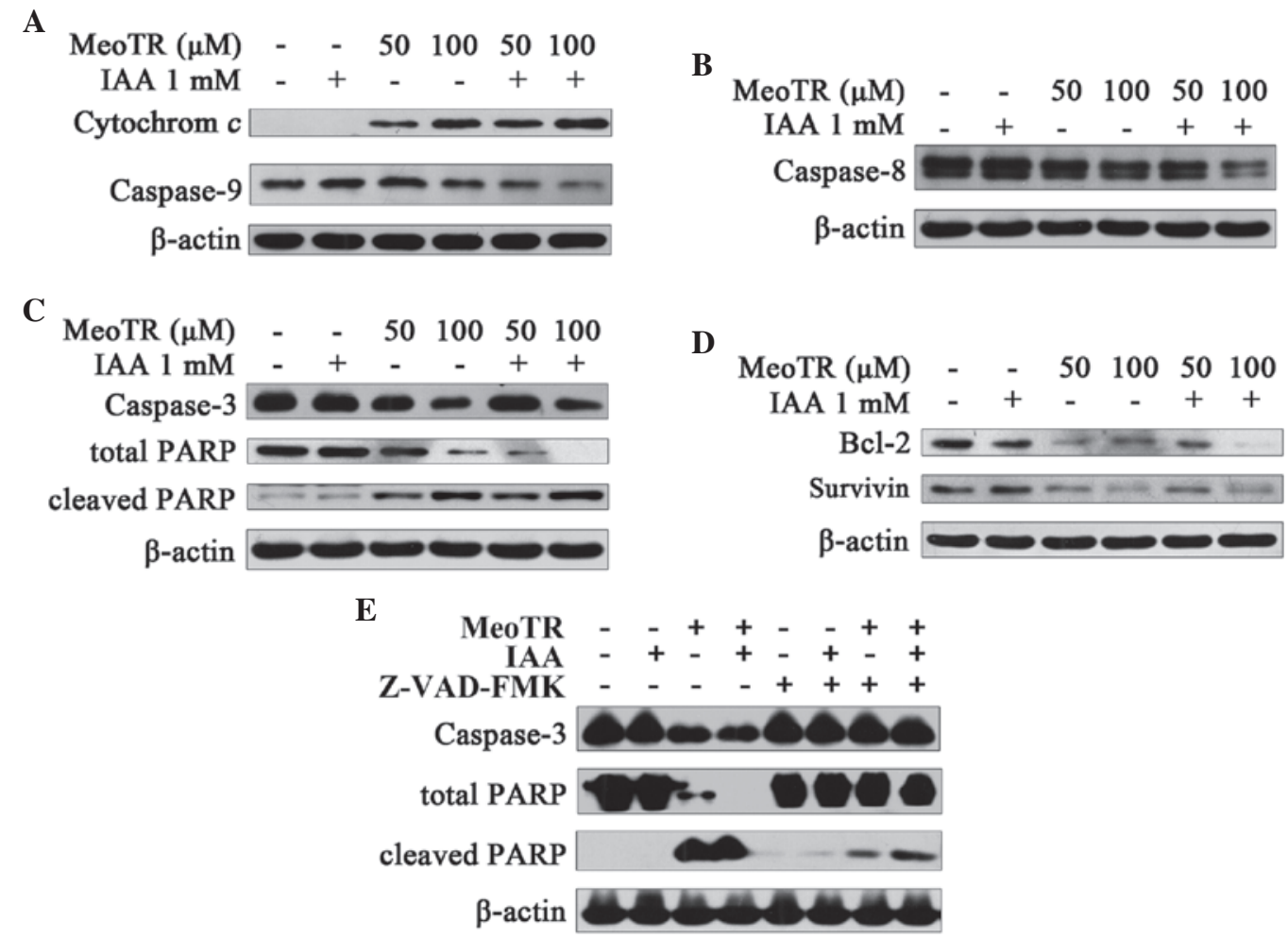

Figure 4. Effect of MeoTR and/or IAA on caspase-dependent apoptosis pathway biomarker protein levels in HeLa cells. HeLa cells were incubated with MeoTR (50 and $100 \mu \mathrm{M})$ and/or IAA $(1 \mathrm{mM})$ for $48 \mathrm{~h}$, and then lysed for western blot analysis using specific antibodies. $\beta$-actin was used as an internal protein loading control. Western blotting analysis of: (A) Release of cytochrome $c$ to the cytosol from mitochondria and the activation of caspase-9; (B) effect of MeoTR and/or IAA on the activation of caspase-8; (C) activation levels of caspase-3 and PARP; (D) expression of Bcl-2 and survivin; and (E) effects of MeoTR and/or IAA on caspase-3 activation and PARP cleavage in cells pre-treated with the pan-caspase inhibitor Z-VAD-FMK (50 $\mu \mathrm{M})$ for $1 \mathrm{~h}$, prior to exposure to MeoTR $(50 \mu \mathrm{M})$ and/or IAA $(1 \mathrm{mM})$ in the presence or absence of Z-VAD-FMK for a further $48 \mathrm{~h}$. MeoTR, ortho-methoxytopolin-riboside; IAA, indole-3-acetic acid; PARP, poly-adenosine diphosphate-ribose polymerase; Bcl-2, B cell lymphoma 2; Z-VAD-FMK, Z-Val-Ala-Asp-fluoromethylketone.

whereas combination treatment with MeoTR and IAA was more demonstrated a more potent effect compared with that of MeoTR alone (Fig. 4A). Caspase-8 is the key initiator caspase in the extrinsic caspase-dependent pathway and is activated by auto-phosphorylation $(21,22)$. Treatment with MeoTR alone (Fig. 3B) or in combination with IAA (Fig. 4B) decreased the expression of the caspase- 8 zymogen in a dose-dependent manner; in addition, the combination treatment was shown to accelerate the auto-phosphorylation of caspase-8, whereas treatment with IAA alone had no effect (Fig. 4B).

MeoTR induces the activation of caspase-3, induces the cleavage of PARP and downregulates anti-apoptotic 
A
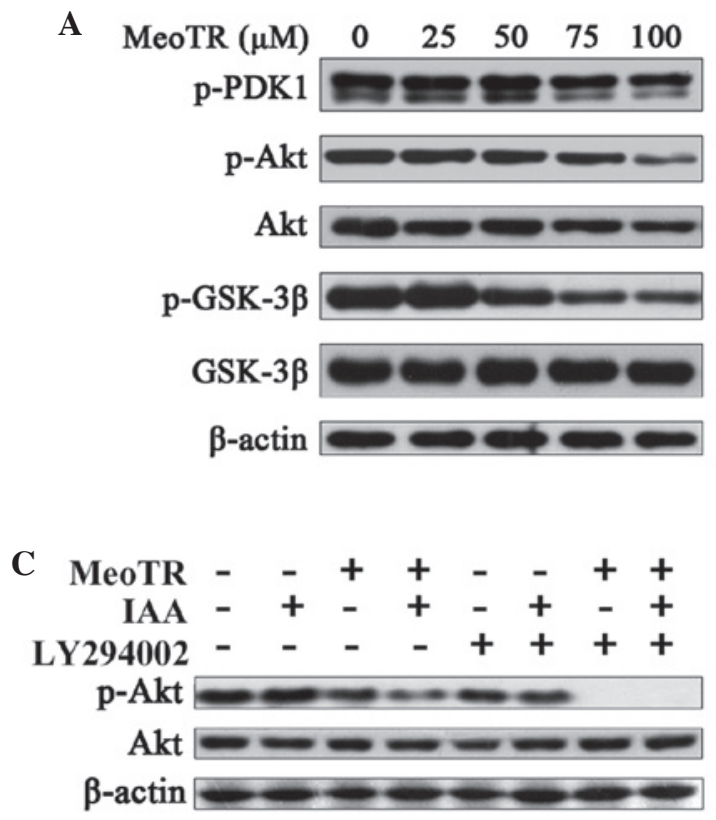
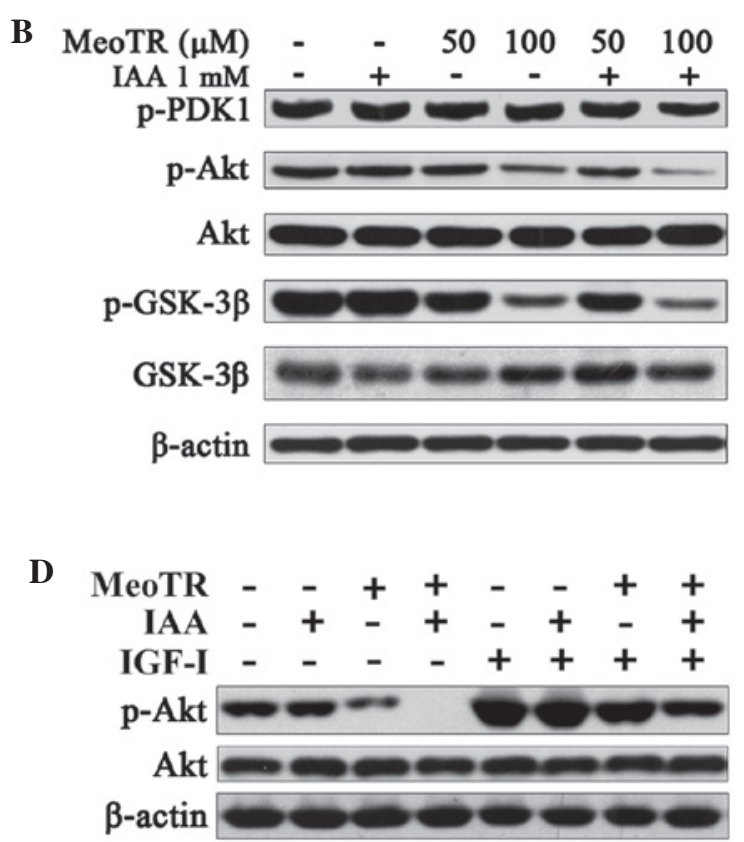

Figure 5. Effect of MeoTR and/or IAA on the Akt signaling pathway in HeLa cells. Following treatment with MeoTR $(25,50,75$ and $100 \mu \mathrm{M})$ alone or the combination of MeoTR $(50$ and $100 \mu \mathrm{M})$ with IAA $(1 \mathrm{mM})$ for $48 \mathrm{~h}$, HeLa cells were lysed for western blot analysis using specific antibodies. $\beta$-actin was used as an internal protein loading control. Western blot analysis of: (A) Effect of MeoTR on the Akt signaling pathway; (B) effect of MeoTR alone or in combination with IAA on the Akt signaling pathway; (C) effects of MeoTR and/or IAA on Akt phosphorylation in the presence of a Akt inhibitor LY294002. HeLa cells were pretreated with LY294002 $(20 \mu \mathrm{M})$ for $30 \mathrm{~min}$, and exposed to MeoTR $(50 \mu \mathrm{M})$ and/or IAA (1 mM) in the presence or absence of LY294002 for a further $48 \mathrm{~h}$; and (D) effects of MeoTR and/or IAA on IGF-I stimulated phosphorylation of Akt. HeLa cells were pretreated with MeoTR (50 $\mu \mathrm{M})$ and/or IAA ( $1 \mathrm{mM}$ ) in serum-free RPMI-1640 medium for $48 \mathrm{~h}$, and stimulated with IGF-I (10 nM) for a further 30 min. MeoTR, ortho-methoxytopolin-riboside; IAA, indole-3-acetic acid; PDK1, pyruvate dehydrogenase kinase 1; GSK-3 $\beta$, glycogen synthase kinase 3 $\beta$; p-, phosphorylated; IGF-1, insulin-like growth factor 1 .

proteins, and IAA potentiates this effect. PARP is the substrate of caspase-3, the executor of apoptosis and a marker of apoptosis. As shown in Fig. 3C, decreased pro-caspase-3 and increased cleaved PARP levels were observed in HeLa cells exposed to different concentrations of MeoTR $(25,50$, 75 and $100 \mu \mathrm{M})$ in a dose-dependent manner, and this effect was enhanced when cells were treated with MeoTR in combination with $1 \mathrm{mM}$ IAA (Fig. 4C), whereas IAA alone did not affect the cleavage of pro-caspase-3 and PARP (Fig. 4C).

In order to further confirm whether caspase cascade activation is involved in the apoptotic mechanism of MeoTR in combination with IAA, HeLa cells were pre-treated with $50 \mu \mathrm{M}$ Z-VAD-FMK, a pan-specific-caspase-inhibitor (23) for $1 \mathrm{~h}$, and exposed to $50 \mu \mathrm{M}$ MeoTR and/or $1 \mathrm{mM}$ IAA in the presence or absence of Z-VAD-FMK for a further $48 \mathrm{~h}$. The MeoTR and/or IAA-mediated activation of caspase-3, decrease in total PARP and increase in cleaved PARP were found to be partially, but not completely, antagonized in the presence of Z-VAD-FMK; in addition, Z-VAD-FMK had no effect on IAA-only treatment (Fig. 4E). These results indicated that MeoTR induced apoptosis in HeLa, which was enhanced by IAA, the mechanism of which may be associated with caspase activation.

Treatment of HeLa cells with increasing concentrations of MeoTR significantly decreased the levels of the anti-apoptotic proteins Bcl-2 and survivin (Fig. 3D), the effect of which was potentiated by combination treatment with IAA (Fig. 4D). Treatment of HeLa cells with $1 \mathrm{mM}$ IAA alone downregulated $\mathrm{Bcl}-2$, whereas survivin expression remained unchanged (Fig. 4D).
MeoTR blocks the Akt signaling pathway and IAA potentiates this effect. In order to identify the signaling pathways involved in MeoTR-mediated apoptosis in HeLa cells, the Akt signaling pathway was investigated in HeLa cells following exposure to MeoTR in the presence or absence of IAA. As shown in Fig. 5A and B, Akt phosphorylation was inhibited in HeLa cells treated with MeoTR in a dose-dependent manner, the inhibitory effect of which was enhanced by combination treatment with IAA, whereas IAA alone had no effect on Akt phosphorylation. GSK-3 $\beta$ is a tumor suppressor gene regulated by the Akt signaling pathway, which is inactivated through phosphorylation (24-26). MeoTR was found to strongly inhibit the phosphorylation of GSK-3 $\beta$ in a dose-dependent manner, the effect of which was enhanced by IAA (Fig. 5A and B), therefore protecting its tumor suppressor function.

In order to further confirm whether Akt inhibition was involved in this mechanism of MeoTR-mediated apoptosis,

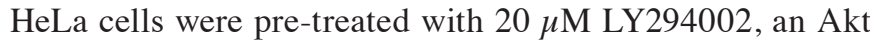
inhibitor (27) for $30 \mathrm{~min}$ and exposed to $50 \mu \mathrm{M}$ MeoTR and/or 1 mM IAA in the presence or absence of LY294002 for a further $48 \mathrm{~h}$. As shown in Fig. 5C, pre-treatment with LY294002 significantly augmented the inhibitory effects of MeoTR on Akt phosphorylation, alone and in combination with IAA. In addition, following treatment with $50 \mu \mathrm{M}$ MeoTR and/or $1 \mathrm{mM}$ IAA in serum-free RPMI-1640 medium for $48 \mathrm{~h}$, HeLa cells were stimulated by $10 \mathrm{nM}$ IGF-I, an Akt activation factor (28) for $30 \mathrm{~min}$. MeoTR alone, as well as combination with IAA, significantly inhibited the IGF-I-stimulated Akt activation (Fig. 5D), therefore further confirming the inhibitory effect of MeoTR on the Akt 
A

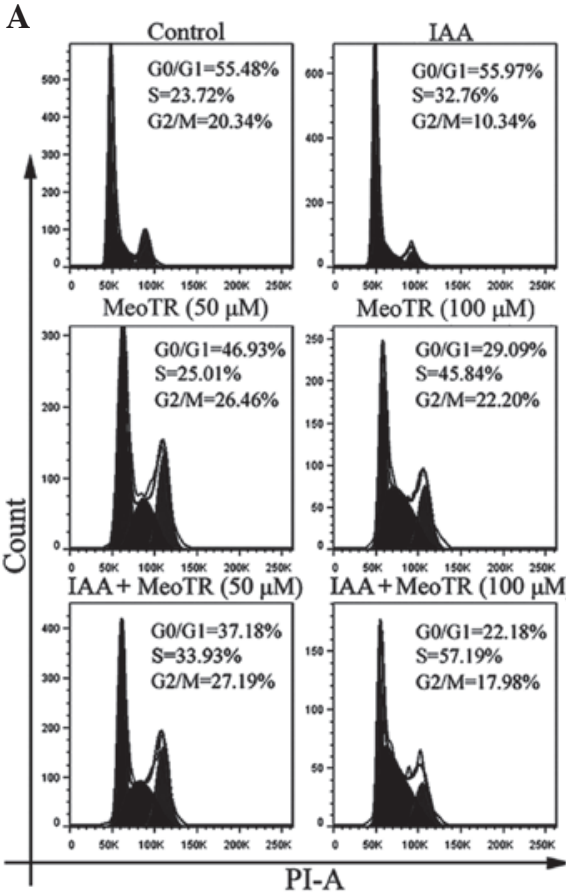

B

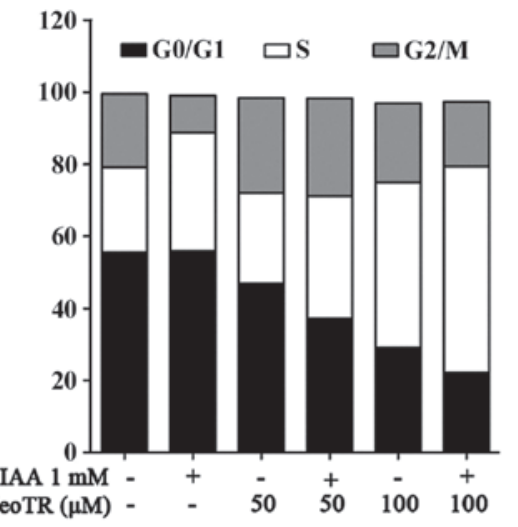

C

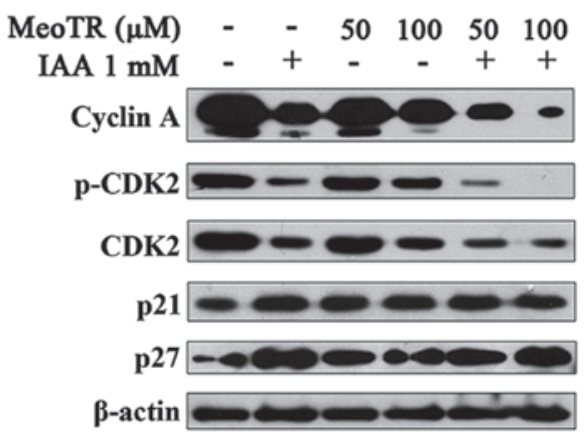

Figure 6. Effects of MeoTR and/or IAA on cell cycle progression arrest. (A and B) Effect of MeoTR (50 and $100 \mu \mathrm{M})$ and/or $1 \mathrm{mM}$ IAA on cell cycle progression arrest, as determined using flow cytometric analysis with PI staining. The two peaks represents cells in $\mathrm{G}_{1}$ (the first) and $\mathrm{G}_{2} / \mathrm{M}_{\text {(the second) }}$ phase, and the area between them represents the S phase. (C) Effect of IAA and/or MeoTR on the expression of cyclin A, CDK2, p-CDK2, p21 and p27 in HeLa cells. Cells were pre-treated with MeoTR $(50$ and $100 \mu \mathrm{M})$ and/or IAA $(1 \mathrm{mM})$ for $48 \mathrm{~h}$, and or lysed for western blot analysis using specific antibodies. $\beta$-actin was used as an internal protein loading control. MeoTR, ortho-methoxytopolin-riboside; IAA, indole-3-acetic acid; CDK2, cyclin dependent kinase 2; p-, phosphorylated; PI, propidium iodide.

pathway, the effect of which was enhanced by combination treatment with IAA.

IAA enhances the inhibitory effect of MeoTR on HeLa cells through arresting cell cycle progression and accumulating in $S$-phase. In order to investigate the potential synergistic inhibitory mechanism of MeoTR and IAA on HeLa cells, HeLa cells were treated with MeoTR (50 and $100 \mu \mathrm{M})$ and/or $1 \mathrm{mM}$ IAA for $48 \mathrm{~h}$ and examined using flow cytometric analysis. MeoTR and IAA induced a marked accumulation of cells in S-phase, as indicated by the increased proportion of cells in S-phase, which was further enhanced by combination treatment with IAA. In addition, the effect of MeoTR alone or in combination with IAA cell cycle progression occurred in a dose-dependent manner (Fig. 6A and B).

The complex of cyclin A and CDK2 has a key role in the progression of cells from S-phase into G2/M-phase, and threonine phosphorylation of CDKs has been reported to enhance the binding of cyclins $(29,30)$. As shown in Fig. 6C, treatment of HeLa cells with IAA or MeoTR, independently, attenuated the expression of cyclin A as well as that of total and p-CDK2; in addition, the combination treatment resulted in a more potent inhibitory effect. IAA and/or MeoTR treatment also resulted in a significant increase in p21 and p27 expression levels (Fig. 6C), which were previously reported to inhibit the formation of CDK-cyclin complexes in order to negatively regulate the cell division cycle (31). Overall, these results indicated that IAA synergistically enhanced MeoTR-induced anti-proliferative and apoptotic effects.

\section{Discussion}

MeoTR is a member of the aromatic cytokinin family. The results of the present study showed that MeoTR had a marked cytotoxic effect on HeLa cells (Fig. 1B), while combination treatment with IAA enhanced the effect of MeoTR (Fig. 1D). The effect of the combination treatment was further clarified through the calculation of CDI (Table I).

Apoptosis is a physiological process that is critical for animal development and tissue homeostasis and is characterized by distinct morphological as well as biochemical features $(32,33)$. In addition, two caspase-dependent pathways have been well characterized (34), which are important for cell death induced by apoptosis (35). The intrinsic caspase-dependent pathway is mediated by the release of cytochrome $c$ from mitochondria into the cytosol (20), where it promotes the interaction of apoptosis protein-activating factor 1 (APAF-1) and pro-caspase-9, leading to the formation of the apoptosome, which in turn results in the activation of the initiator caspase-9 (21-36). In the extrinsic caspase-dependent pathway, caspase- 8 is the key initiator caspase $(21,22)$. The activation of the extrinsic pathway is 
divided into two steps as follows: First, cell surface death receptors engage with their specific ligands, including CD95, tumor necrosis factor (TNF) receptor or TNF-related apoptosis-inducing ligand receptor, which results in the assembly of the death-inducing signal complex (DISC), allowing caspase- 8 to reach a sufficient local concentration of zymogen and enzymatic activity; and secondly, the authophosphorylation and activation of caspase- 8 triggers its separation from DISC, which enables it to access other substrates $(37,38)$. Once the initiator caspases are activated by either the intrinsic or extrinsic signaling pathway, they trigger the activation of the effector caspases-3/7 and the cleavage of PARP, leading to the rapid and irreversible morphological and biochemical changes of apoptosis (39).

In the present study, MeoTR treatment was found to increase the content of cytochrome $c$ in the cytosol of HeLa cells, indicating the release of cytochrome $c$ from mitochondria into the cytosol, and caspase-9 was activated (Fig. 3A). In addition, the activation of the initiator caspase- 8 was detected (Fig. 3B). These results suggested that MeoTR-induced apoptosis in HeLa cells was mediated by the intrinsic and extrinsic caspase-dependent pathways. In cells exposed to combination treatment, the initiator proteins of the two pathways, caspase- 9 and caspase- 8 , were activated and the degree of activation was increased (Fig. 4A and B); however, IAA alone had no effect on the activation of these proteins or apoptosis (Fig. 4A,B, 2D). These data further illustrate that the enhancement effect of IAA on MeoTR was not a simple superposition of cytotoxicity.

The intrinsic and extrinsic pathways are subject to negative regulation by numerous anti-apoptotic proteins, including Bcl-2 (40) and survivin $(41,42)$. In the present study, when used independently, MeoTR downregulated these anti-apoptotic proteins, whereas IAA had no observable effect, with the exception of Bcl-2, which was negatively regulated by IAA (Fig. 3D and 4D). Combination treatment with MeoTR and IAA exhibited a more potent effect on reducing the expression of these anti-apoptotic proteins compared with that of each agent alone (Fig. 4D). Therefore, downregulation of the expression of Bcl-2 may be one of the mechanisms underlying the potentiating effect of IAA on MeoTR-induced apoptosis.

The Akt pathway has a fundamental role in cell survival and proliferation, and is a potentially effective target for the development of novel drugs for the treatment of malignant cancers (43-45). GSK-3 $\beta$, a pro-apoptotic protein $(24,25)$, acts downstream of the Akt pathway and upstream of caspase-8, and it is inactivated through phosphorylation at serine 9 mediated by the Akt pathway (26). As shown in Fig. 5A in the present study, MeoTR alone attenuated the phosphorylation of Akt in a dose-dependent manner in HeLa cells. In addition, MeoTR and IAA combination treatment exhibited a more potent inhibitory effect on the phosphorylation of Akt compared with that of MeoTR alone (Fig. 5B), although IAA alone had no effect (Fig. 5B). The inhibition of Akt phosphorylation by MeoTR alone and in combination with IAA was further confirmed through the employment of specific Akt signal inhibitor LY294002 (Fig. 5C) and the Akt activation factor IGF-I (Fig. 5D). MeoTR inhibited the phosphorylation of GSK-3 $\beta$ (Fig. 5A), thereby protecting its pro-apoptotic effect, and IAA potentiated the inhibitory effect of MeoTR on
GSK-3 $\beta$ phosphorylation or the promotion of dephosphorylation (Fig. 5B). In addition, the MeoTR-mediated inhibition of PDK1, an upstream regulator of the Akt pathway (43), was comparably weak when used independently (Fig. 5A) or in combination with IAA (Fig. 5B), indicating that MeoTR may directly interfere with Akt phosphorylation and block this cell survival-promoting pathway. In summary, the regulation of the Akt pathway and GSK-3 $\beta$ activity by MeoTR and/or IAA may by the mechanism by which MeoTR mediated the suppression of survival and proliferation as well as the induction of apoptosis, and therefore contributed to the enhanced cytotoxicity of combination treatment.

Previous studies have shown that IAA activation by horseradish peroxidase or UVB may be used to investigate its effects on cancer cells, while IAA alone has a weak cytotoxic effect on the cell lines assessed (14-17,46,47). The results of the present study were in agreement with those of previous studies and showed that IAA had no effect on HeLa cell viability and apoptosis induction. However, the results of the present study demonstrated that IAA as well as MeoTR induced significant accumulation of cells in S-phase, inhibited the expression of S-phase-associated cyclin A, CDK2 and p-CDK as well as increased the expression of cell cycle-inhibitory proteins p21 and p27, with enhanced effects under combination treatment. These results suggested that IAA alone may induce significant delay in progression through S-phase (Fig. 6A-C), which may therefore be the mechanism of IAA enhancement on MeoTR-induced growth arrest and apoptosis induction. In plant tissue culture, cytokinin and auxin are often used in combination to control cell division and differentiation; however, their combined effect on animal/human cancer cells in culture has not yet been reported. The results of the present study confirmed that MeoTR and IAA combination treatment exhibited a more potent effect on HeLa cell viability and apoptosis compared with that of MeoTR treatment alone.

In conclusion, the present results indicated that MeoTR exerted a marked anti-tumor effect on human cervical cancer HeLa cells through the induction of apoptosis and blockage of the Akt pathway, the effect of which was enhanced when treated in combination with IAA, while IAA alone had no cytotoxic effects. IAA therefore exhibited a synergistic effect on MeoTR-induced growth inhibition and apoptosis in HeLa cells through cell cycle progression arrest and accumulation in S-phase, coupled with the negative regulation of the $\mathrm{Bcl}-2$. To the best of our knowledge, the present study was the first to demonstrated that cytokinin and auxin combination treatment had a synergistic antitumor effect in vitro, suggesting the potential effectiveness of combining two plant hormone-like substances for the future treatment of malignant cervical cancer. However, further studies, including pre-clinical studies and clinical trials, are required in order to support this proposed therapeutic strategy.

\section{Acknowledgements}

The present study was supported, in part, by a grant from the National Natural Science Foundation of China (No. 31030045). 


\section{References}

1. Skoog F and Miller CO: Chemical regulation of growth and organ formation in plant tissues cultured in vitro. Symp Soc Exp Biol 11: 118-130, 1957.

2. Dolezal K, Popa I, Hauserova E, et al: Preparation, biological activity and endogenous occurrence of N6-benzyladenosines Bioorg Med Chem 15: 3737-3747, 2007.

3. Strnad M: The aromatic cytokinins. Physiol Plantarum 101: 674-688, 1997.

4. Barciszewski J, Massino F and Clark BF: Kinetin-a multiactive molecule. Int J Biol Macromol 40: 182-192, 2007.

5. Horgan R, Hewett EW, Horgan JM, Purse J and Wareing PF: A new cytokinin from Populus x robusta. Phytochemistry 14 1005-1008, 1975.

6. Jones LH, Martinkova H, Strnad M and Hanke DE: Occurrence of aromatic cytokinins in oil palm (Elaeis guineensis Jacq). J Plant Growth Regul 15: 39-49, 1996.

7. Ördög V, Stirk WA, Van Staden J, Novák O and Strnad M Endogenous cytokinins in three genera of microalgae from the chlorophyta1. J Phycol 40: 88-95, 2004

8. Cheong J, Goh D, Yong JW, Tan SN and Ong ES: Inhibitory effect of kinetin riboside in human heptamoa, HepG2. Mol Biosyst 5: 91-98, 2009.

9. Ishii Y, Hori Y, Sakai S and Honma Y: Control of differentiation and apoptosis of human myeloid leukemia cells by cytokinins and cytokinin nucleosides, plant redifferentiation-inducing hormones. Cell Growth Differ 13: 19-26, 2002.

10. Ishii Y, Sakai S and Honma Y: Cytokinin-induced differentiation of human myeloid leukemia HL-60 cells is associated with the formation of nucleotides, but not with incorporation into DNA or RNA. Biochim Biophys Acta 1643: 11-24, 2003.

11. Wang L, Sun C, Wang ZH and Guo GQ: Mechanism of apoptotosis induced by ortho-topolin riboside in human hepatoma cell line SMMC-7721. Food Chem Toxicol 50: 1962-1968, 2012.

12. Castiglioni S, Casati S, Ottria R, Ciuffreda P and Maier JA: N6-isopentenyladenosine and its analogue N6-benzyladenosine induce cell cycle arrest and apoptosis in bladder carcinoma T24 cells. Anticancer Agents Med Chem 13: 672-678, 2013

13. Goldsmith MH: Cellular signaling: new insights into the action of the plant growth hormone auxin. Proc Natl Acad Sci USA 90: 11442-11445, 1993.

14. Kim DS, Jeon SE and Park KC: Oxidation of indole-3-acetic acid by horseradish peroxidase induces apoptosis in G361 human melanoma cells. Cell Signal 16: 81-88, 2004.

15. Kim DS, Kim SY, Jeong YM, et al: Indole-3-acetic acid/horseradish peroxidase-induced apoptosis involves cell surface CD95 (Fas/APO-1) expression. Biol Pharm Bull 29: 1625-1629, 2006.

16. Folkes LK and Wardman P: Enhancing the efficacy of photodynamic cancer therapy by radicals from plant auxin (indole-3-acetic acid). Cancer Res 63: 776-779, 2003.

17. Kim DS, Kim SY, Jeong YM, et al: Light-activated indole-3-acetic acid induces apoptosis in g361 human melanoma cells. Biol Pharm Bull 29: 2404-2409, 2006.

18. Kim SY, Ryu JS, Li H, et al: UVB-activated indole-3-acetic acid induces apoptosis of pc-3 prostate cancer cells. Anticancer Res 30: 4607-4612, 2010

19. Wang D, Wang Z, Tian B, Li X, Li S and Tian Y: Two hour exposure to sodium butyrate sensitizes bladder cancer to anticancer drugs. Int J Urol 15: 435-441, 2008.

20. Vander Heiden MG, Chandel NS, Williamson EK, Schumacker PT and Thompson CB: Bcl-xL regulates the membrane potential and volume homeostasis of mitochondria. Cell 91: 627-637, 1997.

21. Alnemri ES, Livingston DJ, Nicholson DW, et al: Human ICE/CED-3 protease nomenclature. Cell 87: 171, 1996.

22. Hengartner MO: The biochemistry of apoptosis. Nature 407: $770-776,2000$

23. Liu TZ, Cheng JT, Yiin SJ, et al: Isoobtusilactone A induces both caspase-dependent and -independent apoptosis in Hep G2 cells. Food Chem Toxicol 46: 321-327, 2008.

24. Pap M and Cooper GM: Role of glycogen synthase kinase-3 in the phosphatidylinositol 3-Kinase/Akt cell survival pathway. J Biol Chem 273: 19929-19932, 1998.
25. Pap $\mathrm{M}$ and Cooper GM: Role of translation initiation factor $2 \mathrm{~B}$ in control of cell survival by the phosphatidylinositol 3-kinase/Akt/glycogen synthase kinase 3beta signaling pathway. Mol Cell Biol 22: 578-586, 2002.

26. Lin CF, Chen CL, Chiang CW, Jan MS, Huang WC and Lin YS GSK-3beta acts downstream of PP2A and the PI 3-kinase-Akt pathway and upstream of caspase-2 in ceramide-induced mitochondrial apoptosis. J Cell Sci 120: 2935-2943, 2007.

27. Bondar VM, Sweeney-Gotsch B, Andreeff M, Mills GB and McConkey DJ: Inhibition of the phosphatidylinositol 3'-kinase-AKT pathway induces apoptosis in pancreatic carcinoma cells in vitro and in vivo. Mol Cancer Ther 1: 989-997, 2002.

28. Zheng WH and Quirion R: Insulin-like growth factor-1 (IGF-1) induces the activation/phosphorylation of Akt kinase and cAMP response element-binding protein (CREB) by activating different signaling pathways in PC12 cells. BMC Neurosci 7: 51, 2006.

29. Chen T, Stephens PA, Middleton FK and Curtin NJ: Targeting the $\mathrm{S}$ and $\mathrm{G} 2$ checkpoint to treat cancer. Drug Discov Today 17: 194-202, 2012.

30. Vermeulen K, Van Bockstaele DR and Berneman ZN: The cell cycle: a review of regulation, deregulation and therapeutic targets in cancer. Cell Prolif 36: 131-149, 2003.

31. Yoon MK, Mitrea DM, Ou L and Kriwacki RW: Cell cycle regulation by the intrinsically disordered proteins p21 and p27. Biochem Soc Trans 40: 981-988, 2012.

32. Sarraf CE and Bowen ID: Proportions of mitotic and apoptotic cells in a range of untreated experimental tumours. Cell and tissue kinetics 21: 45-49, 1988.

33. Wyllie AH: Apoptosis (the 1992 Frank Rose Memorial Lecture). Br J Cancer 67: 205-208, 1993.

34. Green DR: Apoptotic pathways: paper wraps stone blunts scissors. Cell 102: 1-4, 2000.

35. Scoltock AB and Cidlowski JA: Activation of intrinsic and extrinsic pathways in apoptotic signaling during UV-C-induced death of Jurkat cells: the role of caspase inhibition. Exp Cell Res 297: 212-223, 2004.

36. Li P, Nijhawan D, Budihardjo I, et al: Cytochrome c and dATP-dependent formation of Apaf-1/caspase-9 complex initiates an apoptotic protease cascade. Cell 91: 479-489, 1997.

37. Boatright KM, Renatus M, Scott FL, et al: A unified model for apical caspase activation. Mol Cell 11: 529-541, 2003.

38. Kruyt FA and Schuringa JJ: Apoptosis and cancer stem cells: Implications for apoptosis targeted therapy. Biochem Pharmacol 80: 423-430, 2010.

39. Budihardjo I, Oliver H, Lutter M, Luo X and Wang X: Biochemica pathways of caspase activation during apoptosis. Annu Rev Cell Dev Biol 15: 269-290, 1999.

40. Hockenbery DM, Oltvai ZN, Yin XM, Milliman CL and Korsmeyer SJ: Bcl-2 functions in an antioxidant pathway to prevent apoptosis. Cell 75: 241-251, 1993.

41. Kim E, Matsuse M, Saenko V, et al: Imatinib enhances docetaxel-induced apoptosis through inhibition of nuclear factor-kappaB activation in anaplastic thyroid carcinoma cells. Thyroid 22: 717-724, 2012.

42. Lee R and Collins T: Nuclear factor-kappaB and cell survival: IAPs call for support. Circ Res 88: 262-264, 2001.

43. Brazil DP and Hemmings BA: Ten years of protein kinase B signalling: a hard Akt to follow. Trends Biochem Sci 26: 657-664, 2001

44. Lawlor MA and Alessi DR: PKB/Akt: a key mediator of cell proliferation, survival and insulin responses? J Cell Sci 114: 2903-2910, 2001.

45. Martelli AM, Tazzari PL, Tabellini G, et al: A new selective AKT pharmacological inhibitor reduces resistance to chemotherapeutic drugs, TRAIL, all-trans-retinoic acid and ionizing radiation of human leukemia cells. Leukemia 17: 1794-1805, 2003.

46. Kim SY, Ryu JS, Li H, et al: UVB-activated indole-3-acetic acid induces apoptosis of PC-3 prostate cancer cells. Anticancer Res 30: 4607-4612, 2010.

47. Folkes LK and Wardman P: Oxidative activation of indole-3-acetic acids to cytotoxic species-a potential new role for plant auxins in cancer therapy. Biochem Pharmacol 61: 129-136, 2001. 